\title{
CoviD-19 Harnessing the power of social media to support medical training during COVID-19
}

\author{
Authors: Johnathan Hirniak,, Daniel Huddart, ${ }^{\mathrm{B}}$ Rajiv Sethi, ${ }^{\mathrm{C}}$ Connor Dibblin, ${ }^{\mathrm{D}}$ Gursharun Hayer, ${ }^{\mathrm{E}}$ Biyyam M Rao, \\ Cameron Jenkins, ${ }^{G}$ Benjamin Hueso, ${ }^{H}$ Mohammed E Zaman ${ }^{\mathrm{I}}$ and Sunil Sethi ${ }^{3}$
}

Twitter offers a powerful means to share information, suggest ways to help and highlight useful initiatives during the global COVID-19 pandemic. We describe one successful Twitter campaign focusing on the role of medical students (\#MedStudentCovid), led by the volunteer organisation Becoming A Doctor with support from leaders at the General Medical Council, Health Education England, NHS England and the World Health Organization.

KEYWORDS: Medical students, social media, digital health, collaboration, innovation

DOI: $10.7861 /$ fhj.2020-0027

\section{Background}

COVID-19 has disrupted medical practice. With one in four doctors off sick or in isolation, final year medical students and retired physicians have been invited to join the workforce. Meanwhile, a surge of 'fake news' has nurtured vast misinformation and developed public angst, adding to the strain on the NHS.

Social media is an extremely effective communication tool, although it is frequently both underestimated and criticised by its detractors. It opens possibilities of disseminating best practice with vast public reach, connecting innovators and healthcare professionals internationally, and is an open platform to ask for help or address concerns.

\section{Our campaign}

The Becoming A Doctor team (@Becomingadr), a national volunteer-led organisation, has utilised the power of Twitter with three main aims:

Authors: Amedical student, St George's, University of London, London, UK; ${ }^{B}$ doctor, Imperial College London, London, UK; 'clinical entrepreneur fellow, NHS England and NHS Improvement, UK; Dmedical student, King's College London, London, UK; Emedical student, University of Warwick, Coventry, UK; Fmedical student, University of Liverpool, Liverpool, UK; ' Goundation doctor, Buckinghamshire Healthcare NHS Trust, UK; ${ }^{H}$ medical student, King's College London, London, UK; Idoctor, Imperial College London, London, UK; 'project consultant, @BecomingaDr, UK
> To raise awareness and connect students with existing projects and methods of assisting the NHS.

> To provide clarity on best practice and expectations for students involved in assisting the NHS.

> To clarify uncertainties held by final year medical students in their transition to FY1.

Using the hashtag \#MedStudentCovid, we hosted a Twitter chat by tweeting from the @Becomingadr account and responding to replies and tweets containing \#MedStudentCovid. We connected medical students and healthcare professionals, reaching approximately one million individuals across Europe, North America, Asia and Australia according to Talkwalker, a social media analytics platform. This enabled international feedback with networking and collaboration opportunities.

The campaign featured UK and international examples of medical student contributions to easing the demand placed on healthcare systems by the COVID-19 pandemic. These examples included medical students working as 111 support workers or as healthcare assistants, scribing for healthcare professionals, performing standard procedures on patients not infected with COVID-19, supporting healthcare professionals through non-medical means such as grocery shopping and childcare, correcting online misinformation, and producing local infographics. Since the Twitter discussion, students globally have been using the hashtag \#MedStudentCovid to raise concerns and showcase initiatives offering support during COVID-19.

The initiative was supported by leaders at the General Medical Council, Health Education England, NHS England and the World Health Organization, with representatives of each organisation replying to medical students' concerns regarding safety in the workplace, best practice and volunteer expectations, and addressing the queries of final year medical students transitioning into interim FY1 posts.

Tweets involving common medical student queries (Fig 1) and innovative international practices (Fig 2) are just some of the highlights of the discussion.

\section{Conclusion}

The pandemic emphasises the need to engage and share best practice across health systems, and we urge healthcare students and medical professionals to utilise social media to support their colleagues and each other during the global response to the COVID-19 pandemic. 
Laith Alexander @Lai...·29/03/2020 v Laith here med stu at Cambridge.

Main issues for me: (1) concern about supervision, (2) unclear what is expected of us clinically, (3) worries about how crisis will affect my future training + career choices

Look forward to meeting you all :) \#MedStudentCovid
Q2
七】
O 13
$\uparrow$

Prof Colin R Melville $\ldots \cdot$... 29/03/2020 v

Hi Laith.

Supervision as per Foundation programme Work within foundation competencies Working with others to try and avoid further interruption to progression.

Does that help?

\section{\#MedStudentCovid @BecomingaDr \\ Q1 $\uparrow\urcorner 9$ ○13}

Fig 1. A tweet exchange addressing a common medical student query.

\section{Authors' note and acknowledgments}

All authors are members of the Becoming A Doctor team (@Becomingadr,www.becomingadr.org, outreach@becomingadr.org). Our thanks to Jo Szram, Colin Melville, Chris Lawlor, Michael West, Didier Pittet, Simon Fleming, Greta McLachlan, Kate Burnett, Wayne Kirkham, Laith Alexander and all Twitter chat participants.
Prof Didier Pittet @ @Di... .29/03/2020 v \#MedStudentCovid In Geneva Switzerland 廿 we are currently training medical students to perform swabbing for suspected \#COVID19 individuals We need your help. Check with your local entities on ways you can be involved @Hopitaux_unige



Fig 2. A tweet seeking involvement in a practice innovation.

\section{References}

1 Laith A, @Laithalexander, 29 March 2020.https://twitter.com/ Laithalexander/status/1244308223198363651 [Accessed 28 April 2020].

2 Pittet D, @DidierPittet, 29 March 2020. https://twitter.com/ DidierPittet/status/1244315081988546562 [Accessed 28 April 2020].

Addresses for correspondence: Johnathan Hirniak, St George's, University of London, Cranmer Terrace, Tooting, London, UK, SW17 ORE.

Email: johnathanhirniak@gmail.com 